\title{
COMPARATIVE STUDY OF USG B-MODE AND DOPPLER IN EVALUATION OF OVARIAN MASS LESIONS
}

\begin{tabular}{|l|lll}
\hline Radiodiagnosis &
\end{tabular}

Dr. (Prof.) Alka Professor \& HOD, Department of Radiodiagnosis, M.G.M Medical College \& M.Y Agrawal

\section{Dr. P.S.Tripathi}

Dr. Gaurav Bhandari

\section{Soorya K}

Hospital, Indore

Associate Professor, Department of Radiodiagnosis, M.G.M Medical College \& M.Y Hospital, Indore

Assistant Professor, Department of Radiodiagnosis, M.G.M Medical College \& M.Y Hospital, Indore

Third year PG resident, Department of Radiodiagnosis, M.G.M Medical College \& M.Y Hospital, Indore

Assistant Professor, M.G.M Medical College \& M.Y Hospital, Indore.*Corresponding Author

\section{ABSTRACT}

Background: Ovarian cancer is highly lethal due to delayed detection. Ovarian lesions display a myriad of findings on imaging. Knowledge of these is essential to make a diagnosis or list the differentials. USG remains the mainstay imaging modality in this war on cancer. Aims \& Objectives: To assess the diagnostic accuracy of USG B-mode and doppler in characterisation of ovarian mass lesions. Methods: 103 women with ovarian lesions were evaluated with USG B-mode and doppler. The interpretation was compared with histopathology reports wherever possible. Results: With sensitivity and NPV of $81.82 \%$ and $84.62 \%$ of USG B-mode, it is a good primary imaging modality. By showing an increase in specificity to $94.23 \%$, PPV to $89.29 \%$ and accuracy to $87.06 \%$, the addition of doppler in the evaluation acts as a tool in confirmation of malignancy. Conclusion: With good diagnostic performance, USG proves to be a good primary imaging modality. Also, doppler evaluation forms a promising add-on that can be considered.

\section{KEYWORDS}

Ovarian, Ultrasonography [USG], Doppler

\section{INTRODUCTION}

Ovarian cancer represents one-fourth of all gynaecological malignancies and the third most common gynaecological malignancy. Though only eleventh most common cancer in women and only onetenth as common as breast cancer, it's more lethal. ${ }^{[1]}$ The high mortality is due to delayed detection, late symptom onset and lack of proper screening.

The survival rate falls from $90 \%$ in stage I, $70 \%$ in stage II and $39 \%$ in stage III to $17 \%$ in stage IV. Only $15 \%$ of cases are detected when confined to the primary site. ${ }^{[2]}$ The earliest and accurate characterisation of ovarian lesions is thus warranted for better prognosis and decreased morbidity.

USG is an easily available and cost-effective dynamic imaging modality. It helps in lesion characterisation. With a skillful operator, USG can accurately characterise benign and malignant ovarian lesions, and the lesions requiring further workup.

\section{MATERIALS \& METHODS}

103 patients with ovarian lesions were evaluated in the Department of Radiodiagnosis in M.G.M Medical College and M.Y Hospital, Indore from March 2019 to September 2020 after ethical and scientific committee clearance. Transabdominal and transvaginal USG was done. The lesion was evaluated in terms of its nature [cyst Vs solid], septae, echogenicity, solid portions, papillary projections, laterality, vascularity and ancillary features. Results were compared with the histopathological report. Statistical analysis was done. Cohen's kappa was applied.

\section{RESULT}

The age ranged from 18 to 80 years, with mean of 41.57 years [SD $=13.8$ ]. $32 \%$ were bilateral. $64.1 \%$ were cystic, $33 \%$ were mixed and $2.9 \%$ were solid. $61 \%$ of the lesions with cystic components were multilocular. Among the cystic and mixed lesions, thick septations, papillary projections and solid components were seen in $22 \%, 34 \%$ and $33 \%$ cases respectively. Solid lesions in our study were welldefined, heterogeneous, with foci of calcification, and 1 showing necrosis. The ancillary features - Ascites, omental deposits, lymphadenopathy, liver metastasis and pleural effusion were seen respectively in $21.3 \%, 6.8 \%, 4.9 \%, 2.9 \%$ and $4.8 \%$. Vascularity was seen in $71.8 \%$ cases, with central, septal and peripheral in $13.6 \%$, $14.6 \%$ and $43.6 \%$ respectively. $\mathrm{RI}<0.5$ was observed in $32.4 \%$ of the cases with vascularity. Considering intralesional solid component, papillary projections, thick septations and the noted ancillary features as features suggesting malignant nature, B-mode USG characterised $66 \%$ cases as benign and $34 \%$ as malignant. Adding intralesional low resistance blood flow as a malignant feature, $72.8 \%$ of cases were characterised as benign and $27.2 \%$ as malignant. Histopathological examination was done in 85 cases and $61 \%$ were benign and $39 \%$ were malignant. B-mode USG showed significant sensitivity [81.82\%] and specificity [84.62\%]. There was a notable increase of specificity [94.23\%], PPV [89.29\%] and accuracy [87.06\%] when doppler findings were also considered in the characterisation.

\section{DISCUSSION}

USG continues to be the mainstay imaging modality in the evaluation of ovarian lesions. Considering the burden of ovarian cancer on women's health, early detection is important. With this background, we attempted to characterise the ovarian lesions with USG. The study population ranged from 18 to 80 years old, with a mean age of 41.57 years. More or less equal prevalence was noted in all age groups. Thus, it doesn't spare any age group.

The ovarian lesions we studied were predominantly purely cystic [64.1\%], and the least was solid lesions [2.9\%]. This is comparable to the studies by Guerra et $\mathrm{al}^{[3]}$ in 2008 and Khaled et al ${ }^{[7]}$ in 2014. Similar to the studies done by Umemoto et $\mathrm{al}^{[4]}$ in 2006 and Garg et al ${ }^{[5]}$ in 2017, the majority of the lesions in our study were unilateral [68\%].

Features like intralesional solid component, papillary projections, thick septations, ancillary features and intralesional low resistance vascularity point towards the malignant nature of the lesion. Among cystic and mixed lesions, thick septations, papillary projections and solid components were found in $22 \%, 34 \%$ and $33 \%$ cases respectively. This was comparable with the observation made by Khalaf et $\mathrm{al}^{[6]}$ in 2018, where thick septations, papillary projections and solid components were observed respectively in $20 \%, 32.7 \%$ and $36.6 \%$ of their cases. The solid lesions in our study were well-defined, heterogeneous with foci of calcifications and 1 showing necrosis. This was comparable with the study done by Khaled et $\mathrm{al}^{[7]}$ in 2014 , where 2 out of 20 cases were solid well-defined lesions, with cystic 
degeneration in 1

The ancillary features raising suspicion of malignant nature were noted to be ascites, omental deposits, lymphadenopathy, liver metastasis and pleural effusion, and were found respectively in $21.3 \%, 6.8 \%, 4.9 \%$, $2.9 \%$ and $4.8 \%$ of our cases. This was comparable with the studies done by Guerra et $\mathrm{al}^{[3]}$ in 2008 , who observed peritoneal nodules in $8.9 \%$ and lymphadenopathy in $4 \%$ of their cases. Also, Manali et al ${ }^{[8]}$ in 2014 observed ascites to be the most frequently noted ancillary finding in this setting

On angiogenesis, the newly formed blood vessels show low impedance flow. Thus, low RI could be used as a predictor of ovarian malignancy. $\mathrm{RI}<0.5$ was observed in $32.4 \%$ of the cases in our study. Similarly, Sehgal et al $^{[9]}$ observed a low resistance flow pattern in $21 \%$ of their cases.

On final interpretation, using B-mode morphology, $66 \%$ of the cases were characterised as benign and $34 \%$ as malignant. On adding doppler findings, $72.8 \%$ were characterised as benign and $27.2 \%$ as malignant. This was comparable with the studies done by Garg et $\mathrm{al}^{[5]}$ in 2017 and Khalaf et al ${ }^{[6]}$ in 2018

85 out of 103 lesions were histopathologically evaluated, 8 resolved eventually and 10 were lost during follow-up. Among the histologically evaluated lesions, 61\% were benign and 39\% were malignant. Goyal et $\mathrm{al}^{[10]}$ in 2016 observed a similar result of $60 \%$ benign and $40 \%$ malignant ovarian lesions. Histopathological evaluation revealed 26 mucinous cystadenomas, 16 serous cystadenomas, 4 hemorrhagic cysts, 2 endometrioma, 4 teratoma, 10 serous cystadenocarcinomas and 23 mucinous cystadenocarcinomas. Similar to the studies done by Li et al ${ }^{[11]}$ in 2011 and Khaled et $\mathrm{al}^{[7]}$ in 2014 , the majority of the benign ovarian lesions in our study were epithelial cystadenomas [80\%]. Mucinous cystadenocarcinomas comprise the majority [70\%] of the malignant ovarian lesions. Similarly, Nasr et $\mathrm{al}^{[12]}$ observed $71 \%$ of the malignant lesions to be mucinous cystadenocarcinomas

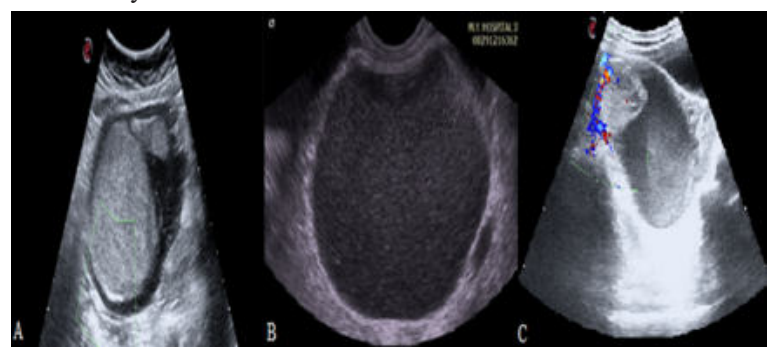

Figure 1: USG findings in A: Fat echogenic content in right ovarian lesion suggestive of teratoma; B: Unilocular right ovarian cystic lesion with ground glass echoes suggestive of endometrioma; $C$ : Multilocular abdominopelvic solid cystic lesion from right ovary with varying echogenicity between locules and central vascularity with $\mathrm{RI}=0.44$, suggestive of mucinous cystadenocarcinoma

By detection of fat content, teratomas were accurately detected on USG, similar to the study by Guerra et $\mathrm{al}^{[3]}$ in 2008 . 1 case was falsely characterised as hemorrhagic cyst, while it turned out to be an endometrioma. The accuracy in the characterisation of serous and mucinous lesions as malignant was respectively noted to be $92 \%$ and $84 \%$ respectively.

Table 1: Statistical analysis of USG evaluation of ovarian lesions

\begin{tabular}{|l|l|l|}
\hline & USG [B-mode] & USG [B-mode + Doppler] \\
\hline Sensitivity [\%] & 81.82 & 75.76 \\
\hline Specificity [\%] & 84.62 & 94.23 \\
\hline PPV [\%] & 77.14 & 89.29 \\
\hline NPV [\%] & 88.00 & 85.96 \\
\hline Accuracy [\%] & 83.53 & 87.06 \\
\hline
\end{tabular}

B-mode USG had significant sensitivity and NPV. On addition of doppler evaluation, there was a notable increase in specificity, PPV and accuracy, however, sensitivity and NPV were reduced. USG [B-mode] had $83.52 \%$ agreement with histopathological examination with cohen's kappa 0.657, [substantial agreement], while USG [B-mode+ Doppler] observed 87.05\% agreement and cohen's kappa of 0.719 [substantial agreement]. This was in concordance with the study by Goyal et $\mathrm{al}^{[11]}$ in 2016 . Hence, by the addition of doppler evaluation, the imaging modality becomes more efficient in true identification of those without the ovarian malignancy.

The area under ROC [Receiver Operating Characteristic] curve was 0.832 for USG B-mode evaluation and became 0.850 when doppler was added, stating better performance when doppler is added
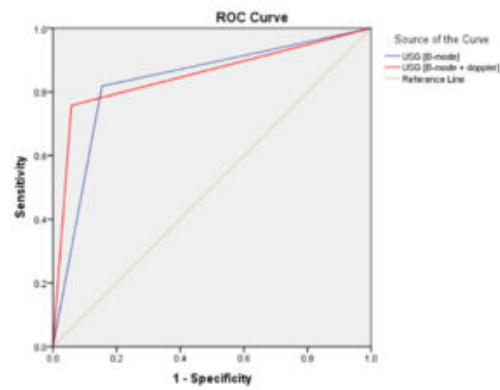

Graph 2: ROC curve

\section{CONCLUSION}

The crucial factor in ovarian malignancy is detection time. With early detection alone, it is possible to significantly improve the survival rate. With a sensitivity and specificity of $81.82 \%$ and $84.62 \%$ respectively, USG is a good primary imaging modality. It is non-invasive, has low cost and is a widely available dynamic imaging modality. On considering doppler findings along with B-mode morphology, specificity improved to $94.23 \%$, and this is a promising add-on that can be utilised, without any additional cost.

\section{REFERENCES}

1. Mehee Choi, Clifton D. Fuller, Charles R. Thomas, Samuel J. Wang. (2008) Conditional survival in ovarian cancer: Results from the SEER dataset 1988-2001

2. Rauh-Hain JA, Krivak TC, Del Carmen MG, Olawaiye AB. Ovarian cancer screenin and early detection in the general population. (2011) Rev Obstet Gynecol. 2011;4(1):15-

3. Guerra A, Cunha TM, Félix A. (2008) Magnetic resonance evaluation of adnexa masses. Acta Radiol. 2008 Jul;49(6):700-9. doi: 10.1080/02841850802064995. PMID: 18568564

4. Umemoto M, Shiota M, Shimono T, et al. (2006) Preoperative diagnosis of ovarian tumors, focusing on the solid area based on diagnostic imaging. J Obstet Gynaecol Res 32:195-201 14

5. Garg S, Kaur A, Mohi JK, Sibia PK, Kaur N. (2017) Evaluation of IOTA Simple Ultrasound Rules to Distinguish Benign and Malignant Ovarian Tumours. J Clin Diagn Res.2017;11(8):TC06-TC09 doi:10.7860/JCDR/2017/26790.10353

6. Khalaf, L.M.R., Desoky, H.H.M., Seifeldein, G.S. et al. (2020) Sonographic and Doppler predictors of malignancy in ovarian lesions. Egypt J Radiol Nucl Med 51, 44 (2020) https://doi org $/ 10.1186 / \mathrm{s} 43055-020-00172-8$

7. Khaled A, Abdrabou A. (2014) The significance of added ADC value to conventional MR imaging in differentiation between benign and malignant ovarian neoplasms. Egyp J Radiol Nuclear Med 2014; 45:997-1002

8. Manali Arora, Vishal Dhirenbhai Thakker, Geetika Sindhwani, Rudra Kant Gogoi: (2017) Ovarian Masses: Hitting the Oncological Dart with Ultrasound and CT - A Comparative Study in a Remote Northeast Indian Town DOI. 10.7860/IJARS/2017/27573:2272

9. Sehgal N. (2019) Efficacy of Color Doppler Ultrasonography in Differentiation of Ovarian Masses. J Midlife Health. 2019;10(1):22-28. doi: 10.4103/jmh. JMH 2318

10. Goyal M, Agarwal VK. (2016) Diagnostic Accuracy of B-mode USG and Doppler Scan for Ovarian Lesions. J Clin Diagn Res. 2016;10(9):TC01-TC04. doi:10.7860/JCDR/2016/20619.8446

11. Li W, Chu C, Cui Y, Zhang P, Zhu M. Diffusion-weighted MRI: a useful technique to discriminate benign versus malignant ovarian surface epithelial tumors with solid and cystic components. (2012) Abdom Imaging. 2012 Oct;37(5):897-903. doi: 10.1007/s00261-011-9814-x. PMID: 22038329

12. Nasr E, Hamed I, Abbas I, Nagat M. (2014) Dynamic contrast enhanced MRI in correlation with diffusion weighted (DWI) MR for characterization of ovarian masses. Egypt J Radiol Nuclear Med 2014; 45:975-985 